\title{
Cutaneous squamous cell carcinoma in a patient with neurofibromatosis type 1: A case report
}

\author{
MITSUAKI ISHIDA and HIDETOSHI OKABE \\ Department of Clinical Laboratory Medicine and Division of Diagnostic Pathology, \\ Shiga University of Medical Science, Otsu, Shiga, Japan \\ Received February 8, 2013; Accepted July 15, 2013
}

DOI: $10.3892 / \mathrm{ol} .2013 .1490$

\begin{abstract}
Neurofibromatosis type 1 (NF1) is an autosomal dominant inherited disease that is characterized by the presence of multiple neurofibromas, café-au-lait spots and iris hamartomas. It is well established that the incidence of tumors in patients with $\mathrm{NF} 1$ is high compared with the normal population and that the majority of the tumors are non-epithelial neoplasms, including neurofibromas, malignant peripheral nerve sheath tumors, gliomas and leukemia. Studies have suggested that patients with NF1 also have a significantly higher risk of certain types of carcinomas. However, the occurrence of cutaneous squamous cell carcinoma (SCC) in a patient with NF1 is extremely rare. The present study describes the second documented case of a cutaneous SCC adjacent to a neurofibroma of the forehead with histopathological analyses in a patient with NF1. An 80 -year-old female with NF1 presented with a rapidly growing skin tumor of the forehead. Histopathological study of the resected forehead tumor demonstrated that there were two tumorous lesions. One was an invasive SCC and the other was a neurofibroma. The lesions were adjacent, but no continuity was present. NF1 is caused by inactivating mutations in the $N F 1$ gene and loss of heterozygosity of this gene has been reported in neurofibromas, malignant peripheral nerve sheath tumors, gliomas and pheochromocytomas in patients with NF1. However, the genetic mechanism of carcinoma development in patients with NF1 is not well understood. Studies have suggested the role of the $N F 1$ and/or the BRCA gene in the occurrence of breast cancer. Additional studies are required to elucidate these mechanisms.
\end{abstract}

\section{Introduction}

Neurofibromatosis type 1 (NF1), also referred to as von Recklinghausen disease, is an autosomal dominant inherited

Correspondence to: Dr Mitsuaki Ishida, Department of Clinical Laboratory Medicine and Division of Diagnostic Pathology, Shiga University of Medical Science, Tsukinowa-cho, Seta, Otsu, Shiga 520-2192, Japan

E-mail: mitsuaki@belle.shiga-med.ac.jp

Key words: neurofibromatosis type 1, neurofibroma, squamous cell carcinoma, skin disease that affects approximately one in 3,000 individuals (1). The disease is characterized by the presence of multiple neurofibromas, café-au-lait spots, iris hamartomas (Lisch nodules) and axillary and inguinal freckling (1). It is well established that the incidence of tumors in patients with NF1 is high compared with the normal population, which is the main reason for the reduced life span of NF1 patients (2). The majority of tumors arising in NF1 patients are neurofibromas, particularly plexiform neurofibromas, which is a hallmark of this disease. Malignant peripheral nerve sheath tumors also affect these patients. Furthermore, patients with NF1 have a greatly increased risk of developing gliomas, leukemia, particularly juvenile myelomonocytic leukemia, pheochromocytoma and rhabdomyosarcoma $(2,3)$. In addition, certain types of carcinomas, including breast cancer, may also occur more frequently in patients with NF1 $(2,4,5)$. However, the occurrence of cutaneous squamous cell carcinoma (SCC) in patients with NF1 has been rarely documented (6). The present study describes a case of an SCC adjacent to a neurofibroma of the forehead in a patient with NF1. Written informed consent was obtained from the patient.

\section{Case report}

Patient. An 80-year-old female with NF1 presented with a rapidly growing skin tumor of the forehead. A physical examination revealed numerous cutaneous nodules across the entire body, which were clinically diagnosed as neurofibromas. The forehead skin tumor was well-circumscribed, dome-shaped with a central keratin plug and adjacent to a neurofibroma. The lesion measured $2.5 \times 2.4 \mathrm{~cm}$ in diameter. Under the clinical diagnosis of keratoacanthoma, a total resection of the forehead tumor with the adjacent neurofibroma was performed.

Methods. The formalin-fixed, paraffin-embedded tissue blocks of the resected skin specimen were cut into $3-\mu \mathrm{m}$ thick sections, deparaffinized and rehydrated. Each section was stained with hematoxylin and eosin and used for immunostaining. Immunohistochemical analyses were performed using an autostainer (XT system BenchMark; Ventana Medical System, Inc., Tucson, AZ, USA) according to the manufacturer's instructions. A mouse monoclonal antibody against Ki-67 (MM1; Novocastra Laboratories, Ltd., Newcastle upon Tyne, UK) and a rabbit polyclonal antibody 
against S-100 protein (Nichirei Bioscience, Tokyo, Japan) were used.

Results. Macroscopically, the cut section of the tumor revealed two tumorous lesions. One was a marked hyperkeratotic tumor invading the upper subcutis and the other was a well-circumscribed nodule in the dermis and subcutis (Fig. 1). Although the two lesions were adjacent, no continuity was noted.

Microscopically, the former tumor revealed papillary proliferation of atypical squamous cells with marked hyperparakeratosis (Fig. 2A). These atypical squamous cells contained large nuclei with coarse chromatin, conspicuous nucleoli and a rich eosinophilic cytoplasm (Fig. 2B). Mitotic figures were frequently observed. The tumor had invaded into the upper subcutis and peritumoral lymphoplasmacytic infiltration was also noted (Fig. 2B). These histopathological features were typical for an invasive SCC. The latter component was a neurofibroma, which was composed of proliferating spindle cells containing bland cigar-shaped nuclei with inconspicuous nucleoli and an eosinophilic cytoplasm (Fig. 2C). No mitotic figures were observed. Immunohistochemically, the spindle cells were diffusely positive for S-100 protein and the $\mathrm{Ki}-67$ labeling index was $<1 \%$. Therefore, this component was diagnosed as a neurofibroma.

\section{Discussion}

Patients with NF1 are at an increased risk of non-epithelial neoplasms of several types, including neurofibromas, malignant peripheral nerve sheath tumors, gliomas, leukemia, pheochromocytoma and rhabdomyosarcoma $(2,3)$. Furthermore, studies have also suggested an increased risk of certain types of carcinomas in patients with NF1 $(2,4,5)$. Seminog and Goldacre (2) recently analyzed 697 cases of carcinomas in 6,739 patients with NF1. The study revealed that patients with NF1 have a significantly high risk of developing carcinomas of the esophagus, stomach, colon, liver, lung, thyroid, breast and ovary (2). Furthermore, a slightly increased risk of non-melanoma skin cancers was reported, though the histopathological subtypes of the skin cancers were not available (2). However, SCC of the skin accounts for only a small number of malignancies in NF1 patients and to the best of our knowledge, only one case of an invasive SCC of the sole of the foot, with analyses of the histopathological features, has been reported in an NF1 patient (6). The present study is the second documented case of an invasive SCC with histopathological analyses in a patient with NF1.

NF1 is caused by inactivating mutations in the NF1 gene, which is located on chromosome 17q11.2. This gene has a tumor suppressor function as the gene product of NF1, neurofibromin, is a major negative regulator of the RAS/ mitogen-activated protein kinase (MAPK) pathway, which transmits mitogenic signals to the nucleus (7). Consistent with Knudson's two-hit hypothesis, NF1 patients with a heterozygous germline NFI mutation develop a somatic mutation in the second wild-type NFI allele, resulting in the development of neurofibromas. The loss of heterozygosity of the $N F 1$ gene is observed in certain types of non-epithelial tumors in patients with NF1. Biallelic NF1 inactivation is observed in neurofibromas and malignant peripheral nerve

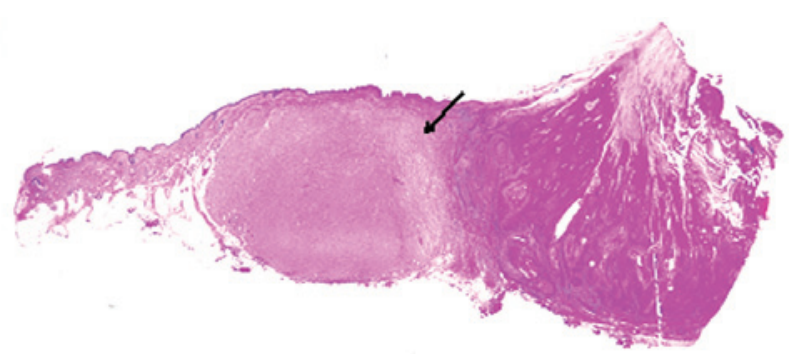

Figure 1. Panoramic view of the forehead tumor. Squamous cell carcinoma (SCC; right) and neurofibroma (left, arrow) are adjacent. However, no continuity is observed (hematoxylin and eosin staining).

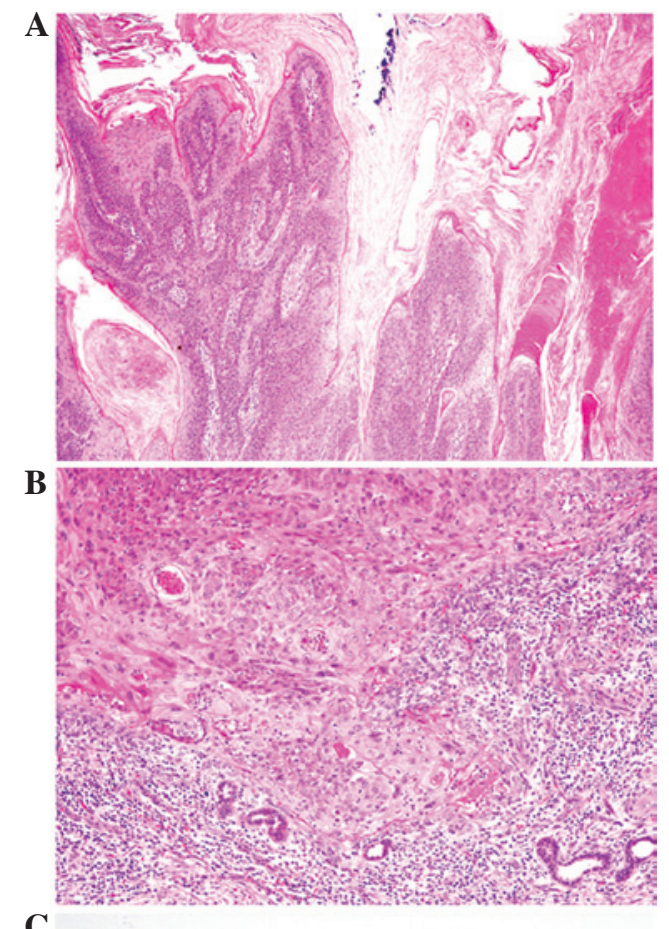

C

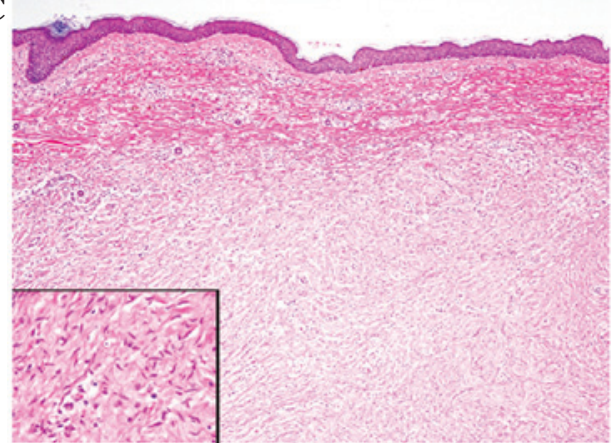

Figure 2. Histopathological observations of the forehead tumor. (A) The SCC is composed of a papillary proliferation of atypical squamous cells with hyperparakeratosis (hematoxylin and eosin staining; magnification, x40). (B) Tumor cells of the SCC have large nuclei with conspicuous nucleoli and a rich eosinophilic cytoplasm. Peritumoral lymphoplasmacytic infiltration is also observed (hematoxylin and eosin staining; magnification, x100). (C) The neurofibroma is composed of proliferating spindle cells. These spindle cells have bland cigar-shaped nuclei (inset) [hematoxylin and eosin staining; magnification, x40 and (inset) x200]. SCC, squamous cell carcinoma.

sheath tumors (8-10). A high proportion of astrocytomas from patients with NF1 also demonstrate a loss of neurofibromin expression and a loss of heterozygosity of the NF1 gene (11). Furthermore, the loss of heterozygosity of the NF1 region has 
been observed in the majority of pheochromocytoma cases in patients with NF1 (12).

The genetic mechanism of carcinoma development in patients with NF1 is not well understood. However, Güran and Safali (13) reported a case of breast carcinoma in an NF1 patient and loss of heterozyosity of the NF1 gene in the carcinoma tissue. BRCA1 or BRCA2 mutations were not observed in this case. Furthermore, Ceccaroni et al (14) reported the cases of five individuals in a family with NF1 who presented with neurofibromas and breast, ovary, peritoneal or rectal carcinomas. The study clearly demonstrated that three individuals shared a common haplotype, including the NFI and BRCAI loci on chromosome 17 and speculated that the occurrence of NF1 and breast carcinoma in this family was due to the presence of two linked mutations at the NFI and BRCAl foci (14).

In conclusion, the present study is the second documented case of cutaneous SCC with an analysis of the histopathological features in a patient with NF1. The increased risk of various types of carcinomas, including non-melanoma skin carcinoma, in patients with NF1 is recognized, however, the molecular mechanism of carcinoma development in patients with NF1 is not well understood. Additional studies are required to clarify this mechanism

\section{References}

1. Brems H, Beert E, de Ravel T and Legius E: Mechanisms in the pathogenesis of malignant tumours in neurofibromatosis type 1 . Lancet Oncol 10: 508-515, 2009

2. Seminog OO and Goldacre MJ: Risk of benign tumours of nervous system, and of malignant neoplasms, in people with neurofibromatosis: population-based record-linkage study. $\mathrm{Br}$ J Cancer 108: 193-198, 2013.

3. Zöller ME, Rembeck B, Odén A, Samuelsson M and Angervall L: Malignant and benign tumors in patients with neurofibromatosis type 1 in a defined Swedish population. Cancer 79: 2125-2131, 1997.
4. Salemis NS, Nakos G, Sambaziotis D and Gourgiotis S: Breast cancer associated with type 1 neurofibromatosis. Breast Cancer 17: 306-309, 2010.

5. Sharif S, Moran A, Houson SM, et al: Women with neurofibromatosis 1 are at a moderately increased risk of developing breast cancer and should be considered for early screening. J Med Genet 44: 481-484, 2007.

6. Friedrich RE, Al-Dam A and Hagel C: Squamous cell carcinoma of the sole of the foot in neurofibromatosis type 1 . Anticancer Res 32: 2165-2168, 2012.

7. Seizinger BR: NF1: a prevalent cause of tumorigenesis in human cancers? Nat Genet 3: 97-99, 1993.

8. Laycock-van Spyk S, Thomas N, Cooper DN and Upadhyaya M: Neurofibromatosis type 1-associtated tumours: their somatic mutational spectrum and pathogenesis. Human Genomics 5: 623-690, 2011

9. Garcia-Linares C, Fernández-Rodriguez J, Terribas E, et al: Dissecting loss of heterozygosity $(\mathrm{LOH})$ in neurofibromatosis type 1-associated neurofibromas: Importance of copy neutral LOH. Hum Mutat 32: 78-90, 2011.

10. Upadhyaya M, Kluwe L, Spurlock G, et al: Germline and somatic NF1 gene mutation spectrum in NF1-associated malignant peripheral nerve sheath tumors (MPNSTs). Hum Mutat 29: 74-82, 2008.

11. Gutmann DH, Donahoe J, Brown T, James CD and Perry A: Loss of neurofibromatosis 1 (NF1) gene expression in NF1-associated pilocytic astrocytomas. Neuropathol Appl Neurobiol 26: 361-367, 2000 .

12. Bausch B, Borozdin W, Mautner VF, et al; European-American Phaeochromocytoma Registry Study Group: Germline NF1 mutational spectra and loss-of-heterozygosity analyses in patients with pheochromocytoma and neurofibromatosis type 1. J Clin Endocrinol Metab 92: 2784-2792, 2007.

13. Güran S and Safali M: A case of neurofibromatosis and breast cancer: loss of heterozygosity of NF1 in breast cancer. Cancer Genet Cytogenet 156: 86-88, 2005.

14. Ceccaroni M, Genuardi M, Legge F, et al: BRCA1-related malignancies in a family presenting with von Recklinghausen's disease. Gynecol Oncol 86: 375-378, 2002. 